\title{
The importance of inter-site coherences in the $x$-ray absorption spectra of mixed-valent systems
}

\author{
Subhra Sen Gupta ${ }^{(a)}$, Hiroki Wadati ${ }^{(b)}$ and G. A. Sawatzky \\ Department of Physics and Astronomy, University of British Columbia, Vancouver, BC V6T 1Z1, Canada.
}

PACS 78.70.Dm - X-ray absorption spectra

PACS 75.30.Mb - Valence fluctuation, Kondo lattice, and heavy-fermion phenomena

PACS 78.20.Bh - Theory, models, and numerical simulation

\begin{abstract}
We study the importance of inter-site coherences and quantum interference effects in the x-ray absorption spectroscopy (XAS) of mixed-valent systems. We demonstrate its importance, first for a simple diatomic mixed-valent molecule to elucidate the physics involved, and finally for mixed-valent Ti oxides, using a model calculation including full spin and orbital multiplicities. Our calculations demonstrate the inefficacy of conventional approaches of describing the XAS from mixed-valent systems as incoherent combinations of XAS from the pure-valent end members, and that multiplets forbidden in the single-site approximation can be reached within a multi-site description. These conclusions play an important role in the interpretation of XAS and resonant $\mathrm{x}$-ray scattering (RXS) in terms of the electronic structure of strongly correlated systems.
\end{abstract}

Introduction. - Strongly correlated electron systems, of which the transition metal (TM) compounds are exemplary, form one of the central themes of present day condensed matter physics. An important feature of these systems is the relatively small spatial extent of their valence $3 d$ wave functions, which form narrow bands of 'widths often matched or overwhelmed by the electronelectron Coulomb interactions, leading to strong corre' lation effects. The physical properties of these systems are largely determined by rather local physics dictated by point group symmetries, Hund's rule coupling of the $d$ electrons and short-range superexchange interactions. XAS along with x-ray magnetic circular (XMCD) and linear (XMLD) dichroism, involving $2 p \rightarrow 3 d$ core-valence transitions on the TM site, has served as an important tool for investigating this local physics, viz. the oxidation states, the local orbital occupancies, spin-state, crystal field symmetry, metal-ligand hybridization, Coulomb correlation effects etc., in these systems [1,2].

Furthermore, recently developed RXS methods where the cross section can be expressed in terms of simple firstorder fundamental XAS spectra [3], can, at least in principle, provide unique information concerning the ordering of spin, charge and orbitals in these systems. How-

\footnotetext{
(a)E-mail : subhra@phas.ubc.ca

(b) Present address : Department of Applied Physics and QPEC, University of Tokyo, Hongo, Tokyo 113-8656, Japan.
}

ever, the information contained in the x-ray energy dependent intensities at superlattice reflections often cannot be accessed, especially for doped systems, because of disagreement with conventionally used single-site cluster like model calculations of the fundamental XAS spectra. In fact this disagreement may point to the importance of intersite coherences playing an important role.

Given the complexity of the problem due to several competing interactions, the inference of ground state properties from the spectral lineshapes requires detailed manybody crystal or ligand field multiplet calculations within a configuration interaction (CI) approach [1]. The success of such local approaches, involving a single central correlated $T M$ site coordinated by a shell of ligand atoms (e.g., oxygen, sulphur etc.), implicitly assumes a pure-valent system with an integral nominal filling per TM site (i.e., before hybridization with the ligands), which ensures that: (1) in the ground state, charge fluctuations involving neighboring TM sites are suppressed by the Hubbard $U$; and more importantly, (2) the locally photo-excited electron in the valence $d$ orbital is strongly bound to the core-hole on the same atom in the final state, by an attractive $2 p-3 d$ Coulomb potential, $Q(\sim 1.2 U)$. This strongly suppresses charge fluctuations involving neighbouring TM sites, since the inter-TM-site effective hopping integrals, $T$, are usually much smaller than $Q$ [4,5]. See fig. 1 (configurations $P 1-P 3)$ for a pictorial representation of the relevant 


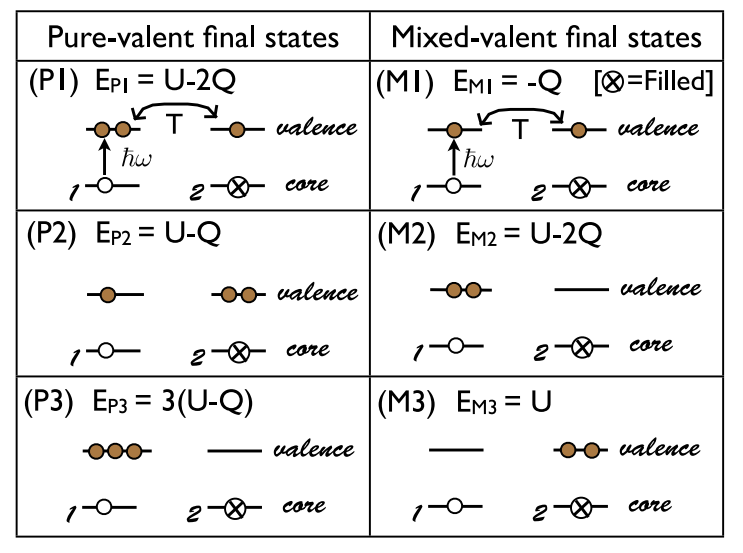

Fig. 1: (colour online) Schematic representing the various relevant final states and their energies, for the XAS of pure-valent $[(P 1)-(P 3)]$ and mixed-valent $[(M 1)-(M 3)]$ correlated systems, in a two-site picture. $U=$ valence electron-electron Coulomb repulsion, $Q=$ core-hole, valence-electron attraction.

physics. The net outcome is that the core-hole and extra valence electron form core-excitonic bound states, so that a single-site description suffices.

However, things become more interesting for mixedvalent or valence fluctuating systems [6. Many of the exotic manifestations of strong correlation e.g., various kinds of charge and spin density wave states, colossal magnetoresistance, high temperature superconductivity etc., arise when the parent compound is hole or electron doped, so that the average nominal number of electrons per TM site is no longer an integer. The interpretation of XAS in these systems is a very important problem, as it can yield important information concerning quantum fluctuations involving also neighboring TM sites, which in turn would serve to explain the observed exotic properties. The general assumption, till now, has been that one can get away with incoherently averaging the spectra (weights determined by the doping) calculated on the basis of single TM site clusters with integral nominal fillings [7. But this approach neglects crucial quantum interference effects arising out of coherent charge fluctuations between different TM sites and may miss some important physics. It implies a phaseseparated scenario instead of uniform mixed-valency. It is the conditions under which these coherent mixing effects play an important role, that we address in this paper.

To treat a doped system, the minimum extension should involve two TM sites with either an odd (mixed-valent) or an even (pure-valent) total number of $d$ electrons, in the dimer, in the starting state. One can then compare the "exact" calculation for such a two-site cluster with the incoherent sum of the component spectra from singlesite clusters, to verify the importance of coherence effects. Fig. 1 compares the basic energetics of the various states involved in the presence of a core-hole on one of the atoms and an additional valence electron, between mixed-valent and pure-valent systems. This demonstrates that while the energy difference between the two lowest energy fi- nal states, which are connected by a hopping integral $T$, is $Q$ for the pure-valent case, it is only $|U-Q|$ for the mixed-valent one. It is easy to see then that for $T$ much smaller than $U$ and $Q$, the mixing of final states shown for the pure-valent case is small and can mostly be neglected. However, for $T \sim|U-Q|$, the mixing in the mixed-valent case can be very large indeed.

A simple model. - To describe the physics involved we start with a simple model system, not complicated by a lot of multiplet structure viz., a fictitious diatomic $\mathrm{Li}_{2}^{+}$ molecule with orbitally non-degenerate (but including spin degeneracies), core $1 s$ and valence $2 s$ levels at each Li site, as shown in fig. 2. This basic model was used previously to describe core-level photoemission from mixed-valent systems, illustrating there the importance of the broken symmetry ansatz. [8]. The core $1 s$ levels are filled with a total of 4 electrons, and the valence $2 s$ levels share one electron in all, in the initial state, corresponding to $50 \%$ doping of the $\mathrm{Li}_{2}$ molecule. We calculate the $1 s \rightarrow 2 s$ excitation spectrum for this system, which contains the same basic physics as an allowed core-to-valence transition in XAS.

The Hamiltonian for this molecule is given by :

$$
\begin{aligned}
\mathcal{H} & =\sum_{i, \sigma} \epsilon_{1 s} c_{1 s}^{i \sigma \dagger} c_{1 s}^{i \sigma}+\sum_{i, \sigma} \epsilon_{2 s} c_{2 s}^{i \sigma \dagger} c_{2 s}^{i \sigma}+\sum_{\sigma}\left(T c_{2 s}^{A \sigma \dagger} c_{2 s}^{B \sigma}+h . c .\right) \\
& -J \sum_{i} \overrightarrow{\sigma_{i}} \cdot \overrightarrow{S_{i}}-Q \sum_{i, \sigma}\left(n_{h}^{1 s}\right)^{i} c_{2 s}^{i \sigma \dagger} c_{2 s}^{i \sigma}+U \sum_{i} n_{2 s}^{i \uparrow} n_{2 s}^{i \downarrow}
\end{aligned}
$$

where, the sum over $i=A, B$ runs over the two $\mathrm{Li}$ sites, and $\sigma=\uparrow, \downarrow$ sums over the two spin projections. $c_{1 s, 2 s}^{i \sigma \dagger}\left(c_{1 s, 2 s}^{i \sigma}\right)$ creates (destroys) an electron at the site $i$ with spin $\sigma$, in the $(1 s, 2 s)$ orbitals, respectively. Above, $n_{2 s}^{i \sigma}=c_{2 s}^{i \sigma \dagger} c_{2 s}^{i \sigma}$ is the number operator for the $2 s$ electrons, $\left(n_{h}^{1 s}\right)^{i}=\left(2-\sum_{\sigma} c_{1 s}^{i \sigma \dagger} c_{1 s}^{i \sigma}\right)$ counts the number of holes in the core $1 s$ level, while $\left(\overrightarrow{S_{i}}\right)$ and $\left(\overrightarrow{\sigma_{i}}\right)$ denote the operators for the core and the valence spins, all at site $i$. In eq. (1) the first two terms refer to the on-site energies of the two orbitals $\left(\epsilon_{2 s}>\epsilon_{1 s}\right)$, which merely shift the transition energy and which we take to be zero. The subsequent terms describe, respectively, the $2 s$ electron inter-site hopping $T$, the Hund's rule like $1 s-2 s$ ferromangetic exchange $J$, the $1 s$-hole, $2 s$-electron on site attraction $Q$, and the $2 s-2 s$ on-site coulomb repulsion $U$. In our convention while $T<0, J, Q$ and $U$ are positive numbers.

In fig. 2 we show the basis configurations involved in the initial and final states, their diagonal energies and the off-diagonal matrix elements resulting from eq. (1), for the initial $\left(\mathcal{H}_{i n i}\right)$ and final $\left(\mathcal{H}_{\text {fin }}\right)$ Hamiltonians. The initial Hilbert space involves two degenerate configurations $\left|G_{1}\right\rangle$ and $\left|G_{2}\right\rangle$, and the $2 s$ hopping, $T$, is the only off-diagonal term. We take the $2 s$ electron to be spin-up. This stabilizes the bonding state, i.e., $\left|\psi_{g}\right\rangle=\frac{1}{\sqrt{2}}\left(\left|G_{1}\right\rangle+\left|G_{2}\right\rangle\right)$, as the ground state for $T<0$. The final Hilbert space is more complex, consisting of five relevant configurations $\left|F_{i}\right\rangle(i=$ $1,5)$ as shown in fig. 2. While $\left|F_{1}\right\rangle$ corresponds to neutral $\mathrm{Li}\left(\mathrm{Li}^{0}\right)$, the configurations $\left|F_{2}\right\rangle$ to $\left|F_{5}\right\rangle$ are obtained from 


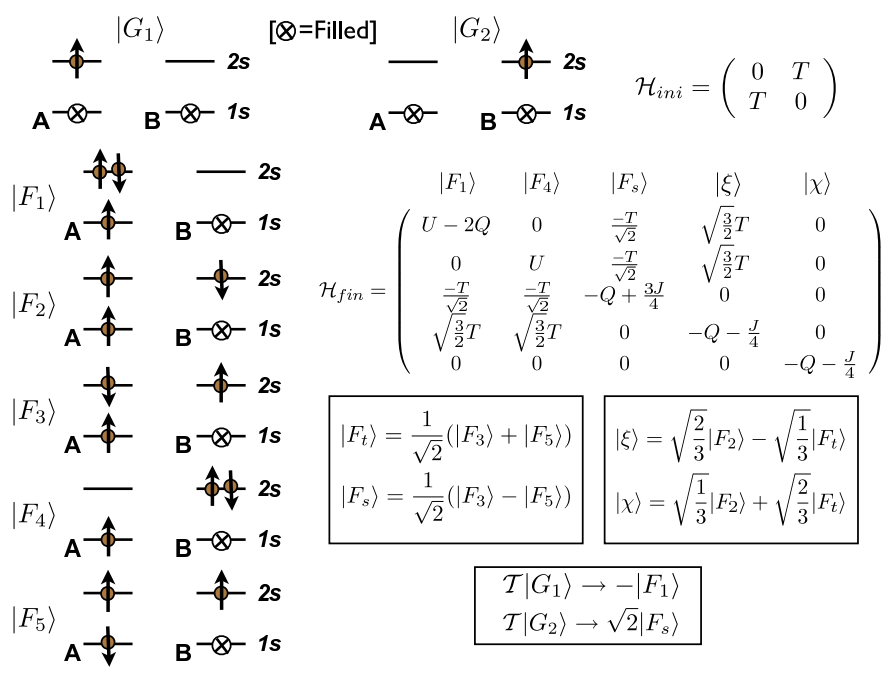

Fig. 2: (colour online) Schematic representation of the various relevant initial $\left(\left|G_{1}\right\rangle-\left|G_{2}\right\rangle\right)$ and final $\left(\left|F_{1}\right\rangle-\left|F_{5}\right\rangle\right)$ configurations (basis states) for the $\mathrm{Li}_{2}^{+}$molecule, shown along with the initial $\left(\mathcal{H}_{\text {ini }}\right)$ and final $\left(\mathcal{H}_{\text {fin }}\right)$ state Hamiltonians. The final state Hamiltonian is written in terms of a transformed basis which is defined below it. The action of the transition operator $(\mathcal{T})$ on the initial basis states is also listed.

the $\mathrm{Li}^{+}$ion, at the core-hole site $A$. The off-diagonal matrix elements now also involve $J$. We have excluded a state composed of the two spin-down $2 s$ electrons and a spinup $1 s$ electron, as it does not connect to any of the other $\left|F_{i}\right\rangle$ configurations. It is instructive to talk in terms of spin states at the core-hole site $A\left(S_{A}\right)$, as well as the total spin $S$ including the effect of the spectator spin doublet at site$B$. In this sense, $\left|F_{1}\right\rangle$ and $\left|F_{4}\right\rangle$ are $|S=1 / 2, m=+1 / 2\rangle$ states derived from the parents $\left|S_{A}=1 / 2, m_{A}=+1 / 2\right\rangle$ and $\left|S_{B}=0, m_{B}=0\right\rangle$. These occur at the energies $(U-$ $2 Q)$ and $U$. The state $\left|F_{2}\right\rangle$, at energy $\left(-Q-\frac{J}{4}\right)$, consists of the triplet state $\left|S_{A}=1, m_{A}=+1\right\rangle$ at site- $A$ and the doublet component $\left|S_{B}=1 / 2, m_{B}=-1 / 2\right\rangle$ at site- $B$. As demonstrated in fig. 2, we combine $\left|F_{3}\right\rangle$ and $\left|F_{5}\right\rangle$ to form singlet $\left(\left|F_{s}\right\rangle\right)$ and triplet $\left(\left|F_{t}\right\rangle\right)$ eigenstates of $S_{A}$. Both $\left|F_{s}\right\rangle$ with $\left|S_{A}=0, m_{A}=0\right\rangle$ at $\left(-Q+\frac{3 J}{4}\right)$, and $\left|F_{t}\right\rangle$ with $\left|S_{A}=1, m_{A}=0\right\rangle$ at $\left(-Q-\frac{J}{4}\right)$ can combine with the $B$-site spin to form total spin doublets. $\left|F_{2}\right\rangle$ being actually the $m_{A}=+1$ component of the site- $A$ triplet, is degenerate with $\left|F_{t}\right\rangle$. As shown fig. 2 these two degenerate triplets can be combined, at $T=0$, into eigenstates of the total spin $S$, to yield the doublet $|\xi\rangle=|S=1 / 2, m=+1 / 2\rangle$ and the quartet $|\chi\rangle=|S=3 / 2, m=+1 / 2\rangle$, both at $\left(-Q-\frac{J}{4}\right)$.

The final state Hamiltonian, $\mathcal{H}_{\text {fin }}$, written in this new basis $\left\{\left|F_{1}\right\rangle,\left|F_{4}\right\rangle,\left|F_{s}\right\rangle,|\xi\rangle,|\chi\rangle\right\}$, now involves only $T$ in its off-diagonal elements, as shown in fig. 22 This is diagonalized to yield the final eigenstates $\left|\psi_{f}^{k}\right\rangle(k=1,5)$. The transition operator for the $1 s \rightarrow 2 s$ excitation is given by :

$$
\mathcal{T}=\mathcal{M} \sum_{\sigma} c_{2 s}^{A \sigma \dagger} c_{1 s}^{A \sigma}
$$

with a spin-independent transition matrix element $\mathcal{M}$. The action of $\mathcal{T}$ on each of the initial basis states is enu- merated in fig. 2, where the negative signs arise because of the antisymmetric nature of the basis configurations, and results in transitions to only those final states that involve a singlet or doublet component at site- $A$. Without loss of generality we set $\mathcal{M}=1$. Then the excitation spectrum is given by the expression :

$$
I(\omega)=\sum_{k}\left|\left\langle\psi_{f}^{k}|\mathcal{T}| \psi_{g}\right\rangle\right|^{2} \delta\left(\omega-\left(E_{f}^{k}-E_{g}\right)\right)
$$

The evolution of the transition energies, $\omega=\left(E_{f}^{k}-\right.$ $\left.E_{g}\right)=\left(E_{f}^{k}-T\right)$, with $T$ is shown in fig. 33(a), for the values $U=0.83 Q$ (i.e., $Q=1.2 U), J=0.2 Q$. All numerical values for eigenenergies (E), hoppings ( $T$ ) etc. for this model are henceforth quoted in units of $Q$. At $T=0$ these correspond to the diagonal energies of $\mathcal{H}_{\text {fin }}$ as discussed above. Now both $\left|F_{s}\right\rangle$ and $\left|F_{t}\right\rangle$ hybridize with $\left\{\left|F_{1}\right\rangle,\left|F_{4}\right\rangle\right\}$ with an amplitude $-\frac{T}{\sqrt{2}}$, while $\left|F_{2}\right\rangle$ hybridizes with the same states with an amplitude $T$. This, together with the definitions in fig. 2, shows that while the doublet $|\xi\rangle$ hybridizes with $\left\{\left|F_{1}\right\rangle,\left|F_{4}\right\rangle\right\}$ with an amplitude $\sqrt{\frac{3}{2}} T$, the quartet state $|\chi\rangle$ is completely non-bonding. This explains why the state $|\chi\rangle$ does not disperse at all with $T$, which shows up as a linear movement of the transition energy, $\omega$, in fig. 3(a), due to the hybridization shift, $T$, of the ground state, while $|\xi\rangle$ disperses only weakly being repelled in opposite directions by $\left|F_{1}\right\rangle$ and $\left|F_{4}\right\rangle$.

Now turning to the trends in the intensities, we find that for $T=0$, the spectra can be visualized as an incoherent combination of spectra from $\mathrm{Li}^{0}$, which involves a doublet to doublet transition $\left(\left|G_{1}\right\rangle \rightarrow\left|F_{1}\right\rangle\right)$ at $(U-2 Q)$, and from $\mathrm{Li}^{+}$which starts from a singlet at site- $A\left(\left|G_{2}\right\rangle\right)$ and hence a transition to only the local singlet, $\left|F_{s}\right\rangle$, at $\left(-Q+\frac{3 J}{4}\right)$ is possible for $T=0$, the triplet $\left|F_{t}\right\rangle$ being forbidden (fig. 3 (b)). These peaks appear in the intensity ratio of $1: 2$, corresponding to just one way of exciting the $\mathrm{Li}^{0}$, compared to the two ways of exciting the $\mathrm{Li}^{+}$ion. For small values of $T$, till about $T=0.05$ as is evident from fig. 3 (b-c), the spectrum basically still has only two peaks with very little intensity in other regions. However, as $T$ increases, there is spectral weight transfer from higher to lower energy so that the two peaks tend towards equal intensities. This could lead to an incorrect conclusion about the actual $2 s$ electron count and, in the case of doped systems, result in incorrect estimates of doping. We also see that the energy splitting between the $\mathrm{Li}^{0}$ and $\mathrm{Li}^{+}$derived states shows very little change up to a critical value of $\left|T_{c}\right| \sim|U-Q| \sim 0.17$ or as long as $|T|<|U-Q|$, and changes almost linearly for $|T|>|U-Q|$. An analysis of these spectra in terms of an incoherent sum of the parent ion spectra would obviously lead to incorrect conclusions regarding both the composition of the material as well as the "chemical" shifts of the core-hole spectra.

Inspection of the unbroadened intensities for finite $T$,

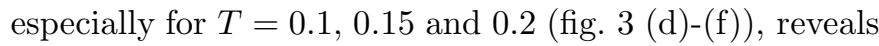
the other crucial fact that for any realistic $T$, transitions 


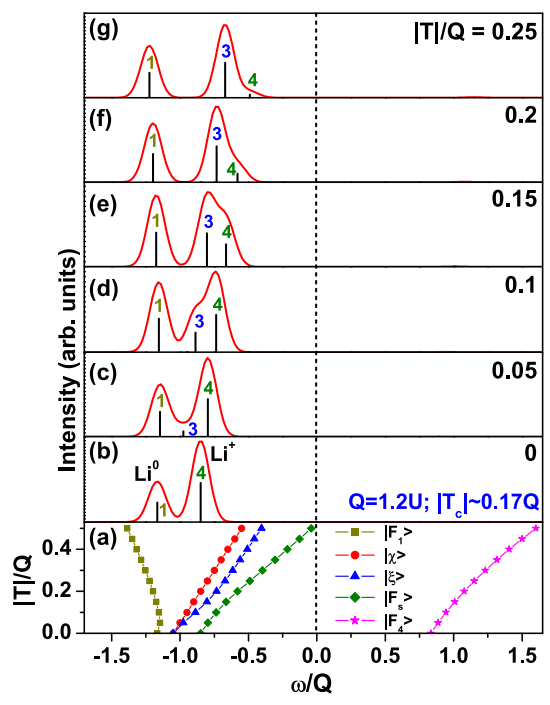

Fig. 3: (colour online) (a) Evolution of the scaled transition energies, $\omega / Q$, to the final eigenstates with the magnitude of the scaled $2 s-2 s$ hybridization, $|T| / Q$, for the $\mathrm{Li}_{2}^{+}$model. The states at $T=0$ from which each eigenstate evolves are marked; (b)-(g) evolution of the $1 s \rightarrow 2 s$ excitation spectrum, with increasing $|T| / Q$, within the same model. Numbers against peaks refer to the final eigenstate from which they originate.

are now possible to previously forbidden (at $T=0)$ multiplets in the $\mathrm{Li}^{+}$-like region, which now shows two peaks with finite intensities that change systematically. This can be understood on the basis that at finite $T$ only the total spin $S$ is relevant. Thus starting from the ground state doublet $|S=1 / 2, m=+1 / 2\rangle$, we can only reach final state doublets, which are the states derived from $\left|F_{1}\right\rangle,\left|F_{4}\right\rangle,\left|F_{s}\right\rangle$ and $|\xi\rangle$, while the quartet $|\chi\rangle$ which does not mix, is still forbidden. So $|\xi\rangle$, which has 0.33 of the $A$-site forbidden triplet $\left|F_{t}\right\rangle$ mixed in at $T=0$, always has a finite contribution from it for finite $T$, demonstrating how forbidden multiplets in a single-site representation now appear as extra structures in the more correct multi-site approach.

Realistic calculations for Ti oxides. - Although this simple model clarifies the basic physics, more realistic calculations including the full orbital and spin degeneracies of a TM ion, multiplet interactions and charge transfer from ligand atoms etc., are needed to approach real correlated systems. To this end we have calculated the isotropic XAS from a mixed-valent $[\mathrm{Ti}-\mathrm{O}-\mathrm{Ti}]$ cluster, mimicking the half-doped oxide $\mathrm{La}_{0.5} \mathrm{Sr}_{0.5} \mathrm{TiO}_{3}$. The purevalent end members $\mathrm{SrTiO}_{3}$ (STO) and $\mathrm{LaTiO}_{3}$ (LTO) consist of $\mathrm{Ti}^{4+}\left(3 d^{0}\right)$ and $\mathrm{Ti}^{3+}\left(3 d^{1}\right)$ ions, respectively, so that the mixed-valent compound has one electron per two Ti atoms. Accordingly our cluster nominally has one electron shared between the two Ti atoms. Alternately, such a mixed-valent bond could also arise at a STO/LTO band-insulator/Mott insulator heterostructure interface, and calculations similar to the present one could help to understand the observed correlated metallic state [7,9] at the interface. Although the use of only a dimer of Ti atoms is a severe approximation to real systems in which there is no dimerization, a calculation with the full coordination of $\mathrm{O}$ and neighbouring $\mathrm{Ti}$ atoms is at the limits of feasability even with modern computers. However, in many real systems in fact, dimer formation seems to occur in particular phases such as in the low temperature insulating phase of $\mathrm{VO}_{2}$ [10, the Zener polaron like scenarios proposed for some Manganites [11, Peierls or spin-Peierls like ground states in quasi one-dimensional systems, to name a few.

Our model calculations include the TM core $2 p$ and valence $3 d$ states as well as the $\mathrm{O} 2 p$ valence states. Full multiplet Coulomb interactions and spin-orbit interaction within the Ti $3 d$ manifold, the spin-orbit interaction within the Ti core $2 p$, and final state core-valence $(2 p-3 d)$ multiplet interactions on the $\mathrm{Ti}$ were taken into account. The relevant spin-orbit parameters and the multipole Coulomb Slater-Condon parameters, $\left(F_{d d}^{2}, F_{d d}^{4}\right)$ and $\left(F_{p d}^{2}, G_{p d}^{1}, G_{p d}^{3}\right)$ were obtained from Cowan's atomic Hartree-Fock code [12, and following the usual convention for TM ions 13 the Slater-Condon parameters were reduced to $80 \%$ of their atomic values. Based on reported values in the literature $[5$ the multiplet averaged $3 d-3 d$ and $2 p-3 d$ Coulomb interactions were set to $U=4.5 \mathrm{eV}$ and $Q=1.2 U=5.4 \mathrm{eV}$, so that $|U-Q|=0.9 \mathrm{eV}$. The Ti $3 d-\mathrm{O} 2 p$ charge transfer energy $(\Delta)$ was defined with respect to a $d^{1}$ system as in LTO, (i.e., $\Delta=E\left(d^{2} \underline{L}\right)-E\left(d^{1}\right)$, where $\underline{L}$ denotes a ligand-hole state) and set to $\Delta=6.0$ $\mathrm{eV}$ [5. An octahedral $\left(O_{h}\right)$ crystal field of magnitude $10 D q=1.5 \mathrm{eV}$ was used on both the Ti sites to effectively take into account the octahedral coordination around the Ti ions, not included in this calculation. The metal-ligand hopping is given by the Slater-Koster parameters $(p d \sigma, p d \pi)$, where $p d \pi=-p d \sigma / 2.2$ was used in all cases [5]. Since our model includes only one ligand oxygen atom explicitly shared between the two Ti atoms, it is quite likely to find more than one hole on the oxygen atom. Thus the usual reasoning that the density of holes on the oxygen atoms is very low and hence the Hubbard $U$ on the $\mathrm{O} 2 p$ is ineffective, no longer holds. So we have also taken into account the full multiplet Coulomb interaction in the $\mathrm{O} 2 p$ manifold with the multiplet averaged interaction $U_{p p}=4.0 \mathrm{eV}$ [14, while $F_{p p}^{2}$ was again obtained from Cowan's code. Calculations were performed for various degrees of covalency, starting from a realistic value of $p d \sigma=-2.5 \mathrm{eV}$ and gradually reducing it through $\{-1.5$, $-0.5,-0.1\} \mathrm{eV}$ to the free ion limit. For the purpose of truncation of the basis set, the convergence of the spectrum was checked as a function of the number of ligand-hole excitations for the largest value of hopping $(p d \sigma=-2.5$ $\mathrm{eV})$, retaining up to 4 ligand-hole $\left(\underline{L}^{4}\right)$ excitations. The spectrum was found to converge well at $\underline{L}^{3}$. Hence the basis was truncated at $\underline{L}^{3}$ for all the calculations, the ones for lower hopping being expected to converge even sooner.

These spectra are to be compared to an incoherent average of single-site pure-valent calculations, to bring out the differences. However an atomic $O_{h}$ crystal field multiplet calculation from a $\mathrm{Ti}^{3+}$ and a $\mathrm{Ti}^{4+}$ ion does not 


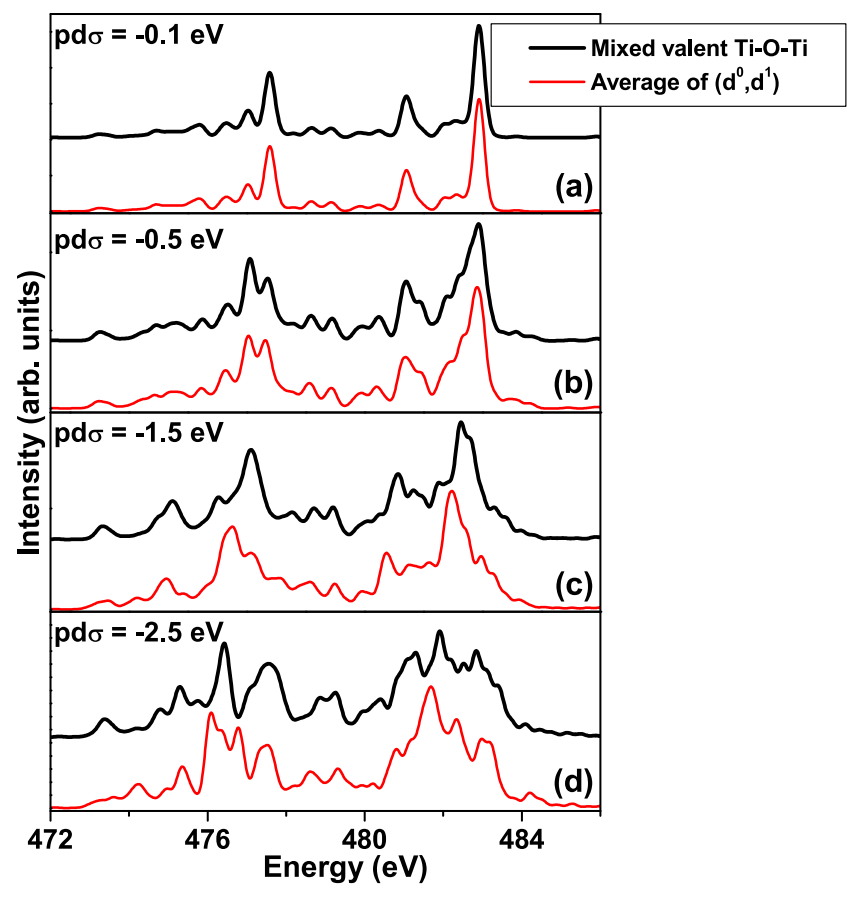

Fig. 4: (colour online) Calculated XAS from a mixed-valent [Ti-O-Ti] cluster (thick line) (mimicking $\mathrm{La}_{0.5} \mathrm{Sr}_{0.5} \mathrm{TiO}_{3}$ ), compared with the incoherently averaged XAS (thin line) from pure-valent $d^{0}$ (mimicking $\mathrm{SrTiO}_{3}: \mathrm{Ti}^{4+}$ ) and $d^{1}$ (mimicking $\left.\mathrm{LaTiO}_{3}: \mathrm{Ti}^{3+}\right)[\mathrm{Ti}-\mathrm{O}]$ clusters, as a function of the metalligand hopping : (a) $p d \sigma=-0.1 \mathrm{eV}$, (b) $p d \sigma=-0.5 \mathrm{eV}$, (c) $p d \sigma=-$ $1.5 \mathrm{eV},(\mathrm{d}) p d \sigma=-2.5 \mathrm{eV}$ (realistic). As the hopping is increased from very small (a) to realistic (d) values, the mixed-valent spectrum deviates farther from its incoherent counterpart.

suffice here, because in choosing a $[\mathrm{Ti}-\mathrm{O}-\mathrm{Ti}]$ cluster, we broke the $O_{h}$ symmetry around the Ti ion via the $\mathrm{Ti}-\mathrm{O}$ bond, and hence we can only compare to a single-site calculation that also breaks the symmetry in the same way. This is achieved by performing calculations for a $\left[\mathrm{Ti}^{3+}-\mathrm{O}\right]$ and a $\left[\mathrm{Ti}^{4+}-\mathrm{O}\right]$ cluster, retaining the $10 D q=1.5 \mathrm{eV} O_{h}$ crystal field, again for the various hoppings as mentioned above. All of the parameters, other than $\Delta$, were kept the same as for the mixed-valent cluster. However, whereas $\Delta=6.0 \mathrm{eV}$ was used for the $\left[\mathrm{Ti}^{3+}-\mathrm{O}\right]$ cluster $\left(3 d^{1}\right)$, an appropriately compensated value of $\Delta^{\prime}=\Delta-U=1.5 \mathrm{eV}$ $\left(\Delta^{\prime}=E\left(d^{1} \underline{L}\right)-E\left(d^{0}\right)\right)$ was used for the $\left[\mathrm{Ti}^{4+}-\mathrm{O}\right]$ cluster $\left(3 d^{0}\right)$, which is the effective charge transfer energy to the $d^{0}$ site within the coherent two-site mixed-valent calculation. For these calculations the full basis set was used without any truncation. A small Gaussian broadening of $\mathrm{FWHM}=0.3 \mathrm{eV}$ was used in all cases.

In fig. (4)(a)-(d) we show the XAS spectra for the mixedvalent cluster (thick black line), starting with the smallest hopping ( $p d \sigma=-0.1 \mathrm{eV}$, atomic-like situation) in panel (a) and then gradually increasing it to the realistic value of $p d \sigma=-2.5 \mathrm{eV}$ in panel (d). In each case we compare it with the spectrum obtained by averaging (thin red line) the XAS from the pure-valent $\left[\mathrm{Ti}^{4+}-\mathrm{O}\right]$ and $\left[\mathrm{Ti}^{3+}-\mathrm{O}\right]$ clusters. Just as in our $\mathrm{Li}_{2}^{+}$calculation, we find that the case for the smallest hopping (a) has almost no coherence

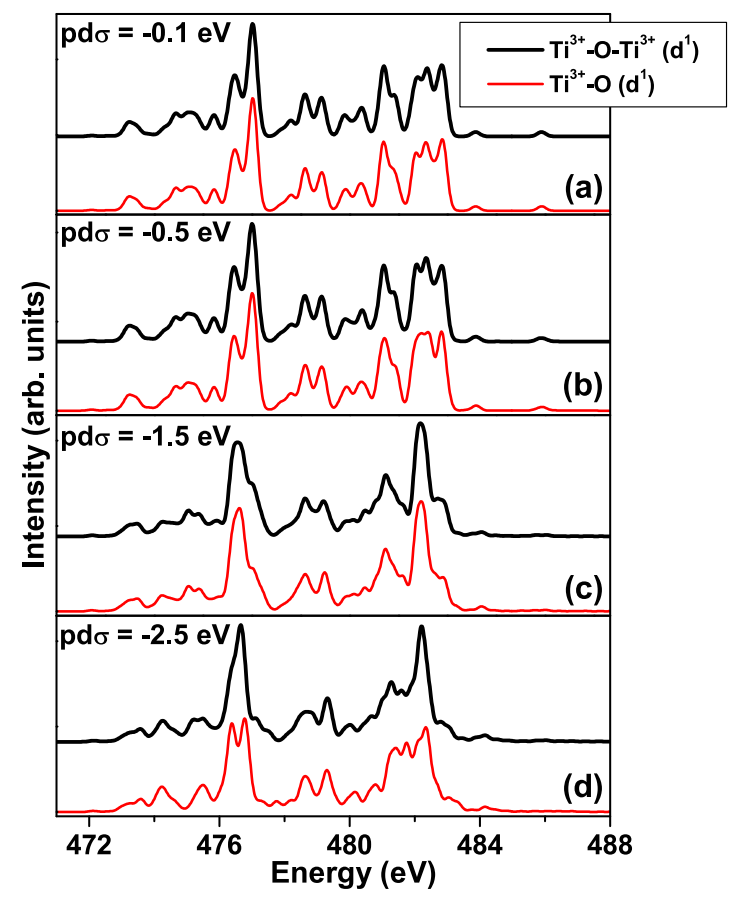

Fig. 5: (colour online) Calculated XAS from a pure-valent $\left(d^{1}\right)\left[\mathrm{Ti}^{3+}-\mathrm{O}-\mathrm{Ti}^{3+}\right]$ cluster (thick line) (mimicking $\mathrm{LaTiO}_{3}$ ) compared with that from a pure-valent $\left[\mathrm{Ti}^{3+}-\mathrm{O}\right]$ cluster $($ thin line), as a function of the metal-ligand hopping : (a) $p d \sigma=-$ $0.1 \mathrm{eV}$, (b) $p d \sigma=-0.5 \mathrm{eV}$, (c) $p d \sigma=-1.5 \mathrm{eV}$, (d) $p d \sigma=-2.5 \mathrm{eV}$ (realistic). Except for the largest hopping $(p d \sigma=-2.5 \mathrm{eV})(\mathrm{d})$, the spectra are nearly identical for all other cases. Even for $p d \sigma=-2.5 \mathrm{eV}$, the differences are much smaller than that for the mixed-valent case (c.f., fig. 4).

between the Ti sites and is formally equivalent to the incoherent average of the XAS from the single site clusters representing the pure-valent end members. However, as we increase the hopping, coherence begins to develop between the two Ti sites and the XAS from the mixed-valent cluster begins to depart from the corresponding incoherent average. While for $p d \sigma=-1.5 \mathrm{eV}$ the differences are quite noticeable, for the more realistic $p d \sigma=-2.5 \mathrm{eV}$, they become truly pronounced. So the single site approximation completely breaks down for realistic hoppings in a mixed-valent system.

Finally, we would like to demonstrate that this problem is much more pronounced in mixed-valent systems compared to pure-valent ones. For the latter, the differences between the two approaches is relatively small, so that one can get away with a single site approximation. To this end we have calculated also the XAS from the two-site pure-valent clusters $\left[\mathrm{Ti}^{4+}-\mathrm{O}-\mathrm{Ti}^{4+}\right]$ and $\left[\mathrm{Ti}^{3+}-\mathrm{O}-\mathrm{Ti}^{3+}\right]$ which are meant to represent the stoichiometric compounds STO and LTO. In accordance with reported values for STO and LTO [5, $\Delta=6.0 \mathrm{eV}$ was used for both calculations. While $\Delta=6.0 \mathrm{eV}$ is a bit on the higher side for STO, the exact value is unimportant and this serves well to bring out the physics involved [15]. All other parameters were exactly the same as used for the mixed-valent 


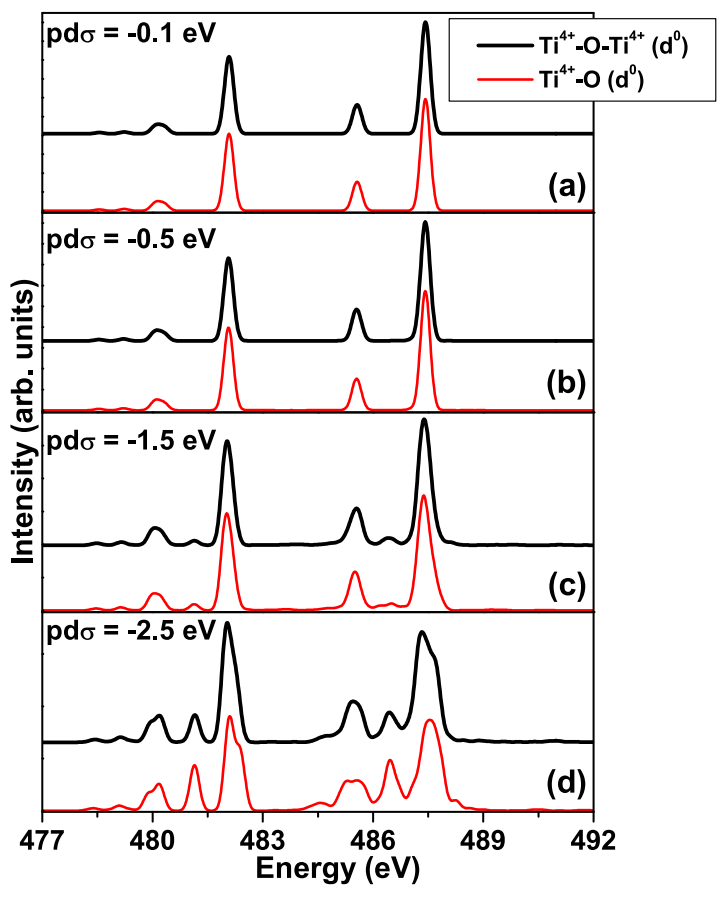

Fig. 6: (colour online) Calculated XAS from a pure-valent $\left(d^{0}\right)$ $\left[\mathrm{Ti}^{4+}-\mathrm{O}-\mathrm{Ti}^{4+}\right]$ cluster (thick line) (mimicking $\mathrm{SrTiO}_{3}[15]$ ) compared with that from a pure-valent $\left[\mathrm{Ti}^{4+}-\mathrm{O}\right]$ cluster (thin line), as a function of the metal-ligand hopping : (a) $p d \sigma=-$ $0.1 \mathrm{eV}$, (b) $p d \sigma=-0.5 \mathrm{eV}$, (c) $p d \sigma=-1.5 \mathrm{eV}$, (d) $p d \sigma=-2.5 \mathrm{eV}$ (realistic). Except for the largest hopping $(p d \sigma=-2.5 \mathrm{eV})(\mathrm{d})$, the spectra are nearly identical for all other cases. Even for $p d \sigma=-2.5 \mathrm{eV}$, the differences are much smaller than that for the mixed-valent case (c.f., fig. 4).

cluster. These were compared to the XAS from the purevalent single-site clusters calculated for the same parameter values. The results are shown, for the $\mathrm{Ti}^{3+}\left(d^{1}\right)$ and $\mathrm{Ti}^{4+}\left(d^{0}\right)$ calculations, in fig. [5 (a)-(d), and fig. 6] (a)-(d), respectively, for the same values of hopping, and shown in the same order as in fig. [4 In each panel the XAS from the two-site pure-valent (thick black line) and single-site purevalent (thin red line) clusters are compared. We find that the only really noticeable differences exist for the largest hopping of $p d \sigma=-2.5 \mathrm{eV}$ (panels (d) in each case), which are much smaller than in the mixed-valent case (fig. 4 (d)).

Conclusion. - Our calculations demonstrate the importance of inter TM site coherences in the XAS of mixedvalent (doped) strongly correlated systems. These effects cause non-trivial differences between the XAS from a truly mixed-valent system and that obtained by incoherently averaging XAS from the corresponding pure-valent end members, whenever the hybridization is comparable to the difference between the valence band Coulomb repulsion $(U)$ and the core-hole potential $(Q)$. This results in strong changes in energy shifts, and a dramatic redistribution of spectral weight away from the statistical limit, between the so-called pure-valent components. It also shows that multiplets forbidden in the single-site approximation can effectively be reached within a multi-site description, for realistic hoppings. For pure-valent systems, this problem is much less severe and the single impurity approximation works reasonably well. This physics has important consequences for doped correlated systems, in general, and TM oxide heterostructures (like LTO/STO) having interfaces across which a change of nominal valency of the TM ion occurs 7, 9. This can also play an important role in distinguishing between charge disproportionation and Zener polaron or dimeron like scenarios [11].

$$
* * *
$$

We would like to acknowledge funding from the Canadian agencies NSERC, CFI and CIFAR. SSG would like to thank Prof. D. D. Sarma for the permission to modify and use the spectroscopy codes developed in his group.

\section{REFERENCES}

[1] de Groot F. M. F. and Kotani A., Core Level Spectroscopy of Solids, edited by D. D. Sarma, G. Kotliar AND Y. Tokura (CRC Press, London, New York) 2008.

[2] de Groot F. M. F., Fuggle J. C., Thole B. T., and Sawatzky G. A., Phys. Rev. B, 42 (1990) 5459; Saitoh T. et al., Phys. Rev. B, 51 (1995) 13942; Thole B. T. and van Der LaAn G., Phys. Rev. B, 38 (1988) 3158; P. Kuiper et al., Phys. Rev. Lett., 62 (1989) 221; Ротze R. H., Sawatzky G. A., and Abbate M., Phys. Rev. B, 51 (1995) 11501; Zabinsky S. I. et al., Phys. Rev. B, 52 (1995) 2995; Thole B. T., Carra P., Sette F., and van Der LaAn G., Phys. Rev. B, 68 (1992) 1943; Carra P., Thole B. T., Altarelli M., and Wang X., Phys. Rev. Lett., 70 (1993) 694; Grazioli C. et al., Phys. Rev. Lett., 95 (2005) 117201; van der LAan G., Arenholz E., Chopdekar R. V., and Suzuki Y., Phys. Rev. B, 77 (2008) 064407.

[3] Haverkort M. W., Hollmann N., Krug I. P., and A. TAnaka, Phys. Rev. B, 82 (2010) 094403; Haverkort M. W., Phys. Rev. Lett., 105 (2010) 167404.

[4] Bocquet A. E. et al., Phys. Rev. B, 46 (1992) 3771.

[5] Saitoh T., Bocquet A. E., Mizokawa T. and FujiMORi A., Phys. Rev. B, 52 (1995) 7934; Bocquet A. E. et al., Phys. Rev. B, 53 (1996) 1161.

[6] Imada M., Fujimori A., and Tokura Y., Rev. Mod. Phys., 70 (1998) 1039; Varma C. M., Rev. Mod. Phys., 48 (1976) 219; Salamon M. B. and Jaime M., Rev. Mod. Phys., 73 (2001) 583; Dagotto E., Rev. Mod. Phys., 66 (1994) 763.

[7] Schüssler-Langeheine C. et al., Phys. Rev. Lett., 95 (2005) 156402; Freeland J. W. et al., condmat/arXiv:1008.1518v1 preprint, (2010) ; WADATI H. et al., J. App. Phys., 106 (2009) 083705; ChakHalian J. et al., cond-mat/arXiv:1008.1373v1 preprint, (2010) ; Оhтоmo A., Muller D. A., Grazul J. L., and Hwang H. Y., Nature, 419 (2002) 378; Wilkins S. B. et al., Phys. Rev. B, 71 (2005) 245102.

[8] Sawatzky G. A. and Lenselink A., J. Chem. Phys., 72 (1980) 3748.

[9] Ishida H. and Liebsch A., Phys. Rev. B, 77 (2008) 115350. 
[10] Goodenough J. B., J. Solid State Chem., 3 (1971) 490.

[11] Daoud-Aladine A. et al., Phys. Rev. Lett., 89 (2002) 097205; Downward L. et al., Phys. Rev. Lett., 95 (2005) 106401; Geck J. et al., New J. Phys., 6 (2004) 152; Bridges F., Downward L., Neumeier J. J. and Tyson T. A., Phys. Rev. B, 81 (2010) 184401.

[12] Cowan R. D., The Theory of Atomic Structure and Spectra (University of California Press, Berkeley and Los Angeles, California.) 1981.

[13] Antonides E., Janse E. C. and Sawatzky G. A., Phys. Rev. B, 15 (1977) 1669; ibid., Phys. Rev. B, 15 (1977) 4596.

[14] GhiJsen J. et al., Phys. Rev. B, 38 (1988) 11322.

[15] The ionic-like spectrum for small $p d \sigma$ (fig. 6 (a)) differs from the experimental $L_{23}$ XAS spectum for STO, since we have not included the large and very disparate broadenings between the two peaks $\left(t_{2 g^{-}}\right.$and $e_{g}$-like) in each of the $L_{2,3}$ regions, arising from lifetime, solid-state dispersional and vibrational effects. See : DE Groot F. M. F., Fuggle J. C., Thole B. T., and Sawatzky G. A., Phys. Rev. B, 41 (1990) 928. 